\title{
Why open research is important for understanding how eye-tracking technology can be used as a tool to evaluate social cognition in intellectual disability
}

Lauren Jenner, 1.jenner@surrey.ac.uk, ORCID: 0000-0003-0841-4884

School of Psychology, University of Surrey, Guildford, Surrey, UK. GU2 7XH.

This work was funded by a studentship from Cerebra and Faculty of Health and Medical Sciences, University of Surrey.

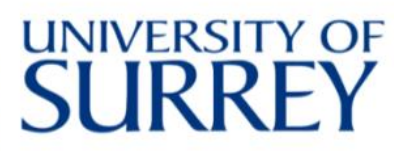

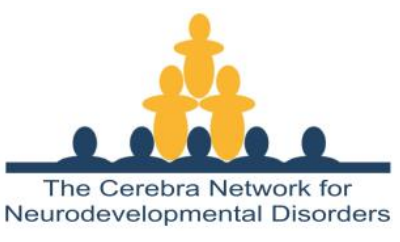

CEREBRA Working wonders for children with brain conditions

This is a pre-publication version of the following article: Jenner, L. (2022). Why open research is important for understanding how eye-tracking technology can be used as a tool to evaluate social cognition in intellectual disability. Developmental Psychology Forum (Issue 95), 7-9. British Psychological Society. 


\section{Why open research is important for understanding how eye-tracking technology can be used as a tool to evaluate social cognition in intellectual disability}

Social functioning is central to the conceptualisation of intellectual disability. Yet, social-cognitive abilities (e.g., emotion discrimination, mental state reasoning) are not well understood. Many traditional social-cognitive measures are demanding on language and domain-general cognitive abilities, meaning they are less suitable and accessible to individuals with an intellectual disability, especially those with moderate to profound impairment. Ultimately, these methodological limitations mean social differences are often understood using parent/caregiver informant measures and/or behavioural observation. A key benefit of eye-tracking technology is that stimuli can be presented in a passive-viewing manner, without the need for explicit responses or verbal demands. Thus, eye-tracking technology has potential to provide information about underlying social-cognitive processes, in a way that is accessible and inclusive of those with varying intellectual and/or verbal abilities. By conducting a systematic review, we aimed to address whether this is the case exploring how eye-tracking technology has been used as a tool to evaluate social cognition among individuals with an intellectual disability. Here, I outline my experience of preregistration, why we felt it was important to include both published and unpublished work, and highlight the need for replication and collaboration, in order to advance this field of research.

When I came to pre-register our systematic review, I realised popular guidance for pre-registering and conducting systematic reviews were not designed for non-interventional research. Items were more focused on intervention and outcome, rather than detailed description of methodology. As the purpose of pre-registration is to facilitate transparency and robustness, whilst constraining reporting bias, I wanted to follow a framework that best suited our review. Luckily as I encountered this issue, the Non-Interventional, Reproducible, and Open Systematic Reviews (NIRO-SR; Topor et al., 2021) guidelines and framework was released - a 68-item checklist supporting planning, pre-registering, and reporting of noninterventional studies in systematic reviews. The pre-print and open repository provide a comprehensive resource, which allowed me to thoroughly plan and pre-register our systematic review. This forward planning not only enabled us to be transparent about our review processes, but made data extraction and synthesis much more straightforward, because I had already defined our research questions, variables of interest, and how I would present the findings before we had started.

We requested unpublished work (e.g., conference proceedings, posters, dissertations, pre-prints, manuscripts under review) through mailing lists (e.g., ID Research UK, COGDEVSOC, Dev-Europe). Though in principle eye-tracking technology is a more accessible measure, there are practical barriers which may influence data collection (e.g., difficulties calibrating/maintaining participant's attention). Data loss can be an issue in any sample, but it is particularly problematic for research on intellectual disability. Participants are harder to recruit, making sample size small, at times underpowered (Farran \& Scerif, 2021), and therefore, less tolerant to missing data. These challenges may mean work does not 
get published. Inclusion of only peer-reviewed studies would overlook the risk of publication bias, and consequently, over-estimate the effectiveness of eye-tracking technology. By including both published and unpublished work, the review represents both the advantages and challenges - providing suggestions for future research.

Whilst reviewing the literature, I noticed that, unsurprisingly, most research was exploratory. When exploratory analyses are used, there is increased potential for Type I error and possibility of sample-dependent results which are less likely to be independently replicated. Of course, exploratory research is important when there is lack of research in an area (and at the time, may make replication seem less important). However, the review highlighted a relatively substantial amount of research; but variability in eye-tracking protocols and heterogeneity of stimuli used.

The goal of many intellectual disability researchers is to improve prognostic and diagnostic methods, identify moderators/mediators, and/or facilitate an evidence-base for more tailored support. Therefore, an important direction for eye-tracking research is sharing and collaborating, in order to strengthen theoretical and clinical implications regarding social cognition. The review emphasises the importance of sharing eye-tracking stimuli to enhance replicability of findings, and more importantly the need to develop a bank of open access, validated stimuli.

We welcome further discussion, and unpublished studies which: 1) include participants with an idiopathic/syndromic intellectual disability, and 2) use eye-tracking technology to record responses to social stimuli and/or a social-cognitive task. Please email 1.jenner@surrey.ac.uk if you are interested. A pre-print of the systematic review will be made available in April. 


\section{References}

Farran, E. K., \& Scerif, G. (2021, May 17). Neurodevelopmental conditions, neuroconstuctivism, and reproducible research; challenges and future directions. https://doi.org/10.31234/osf.io/agwkb

Topor, M., Pickering, J. S., Barbosa Mendes, A., Bishop, D. V. M., Büttner, F. C., Elsherif, M. M., ... Westwood, S. J. (2020, December 14). An integrative framework for planning and conducting Non-Intervention, Reproducible, and Open Systematic Reviews (NIROSR). https://doi.org/10.31222/osf.io/8gu5z 